\title{
The Formation Site of Noninterfacial Misfit Dislocations in InAs/GaAs Quantum Dots
}

\author{
Shuai Zhou, Yumin Liu, Pengfei Lu, Lihong Han, and Zhongyuan Yu \\ State Key Laboratory of Information Photonics and Optical Communications, Institute of Information Photonics and \\ Optical Communications, Beijing University of Posts and Telecommunications, P.O. Box 49 (Room 339), Beijing 100876, China \\ Correspondence should be addressed to Yumin Liu; microliuyumin@hotmail.com
}

Received 30 November 2013; Revised 7 January 2014; Accepted 7 January 2014; Published 16 February 2014

Academic Editor: Wen Lei

Copyright (C) 2014 Shuai Zhou et al. This is an open access article distributed under the Creative Commons Attribution License, which permits unrestricted use, distribution, and reproduction in any medium, provided the original work is properly cited.

Taking elastic anisotropy into consideration, we use a dislocation position dependent model to calculate the preferential formation site of noninterfacial $60^{\circ}$ mixed dislocation segment in ellipsoid shaped InAs/GaAs quantum dots (QDs) which is observed in the experiment. From the result, it is clear that the positions near the right edge of the quantum dot are the energy favorable areas for the noninterfacial $60^{\circ}$ mixed dislocations.

\section{Introduction}

In recent years, InAs/GaAs quantum dots have attracted tremendous attention in the field of nanosemiconductor [17] because of their great potential for device applications. The two most popular methods to fabricate such quantum dots are classical Stranski-Krastanow (S-K) growth [8] and droplet epitaxy growth $[9,10]$. But for both the S-K growth and the droplet epitaxy, with the increasing deposition of InAs on GaAs(001) substrates, the quantum dots begin to release the elastic strain energy by misfit dislocations when their sizes reach a critical value. Because of the harmful effects of dislocations on device performance and reliability, the onset of dislocations in quantum dots must be understood so that devices can be fabricated in fully coherent regimes. The preferential formation sites of interfacial edge and mixed misfit dislocations have been considered theoretically [11]. However, recent reports presented evidence of misfit dislocations located above the island/substrate interface in both S$\mathrm{K}$-grown $\mathrm{GaSb} / \mathrm{GaAs}(001)$ system and droplet epitaxy grown InAs/GaAs(001) quantum dots system $[12,13]$.

In this work, taking elastic anisotropy into consideration, the Finite Element Method (FEM) is used to simulate 3D dislocation position dependent models of noninterfacial dislocated InAs/GaAs quantum dots. The FEM is by far the most widely used numerical technique in science and engineering. It has been proved to be a useful tool in the analysis of the strain and stress state, even for nanoscale materials [14-17]. The stress/strain field and the total elastic energy of InAs/GaAs quantum dots with interfacial and noninterfacial dislocations in different positions are calculated. Comparing the overall energy stored in the quantum dots with dislocations in different positions, we obtain the first nucleation place for the $60^{\circ}$ mixed dislocation in quantum dots.

\section{Model and Method}

The isolated uncapped InAs/GaAs quantum dot heterostructure considered in this paper is shown in Figure 1. The reference point is set to be the left endpoint of the quantum dot in Figure 1. And dislocation positions at the plane perpendicular to the QD growth direction are expressed by "relative distance" $(=L / S)$ which is a ratio of the distance from the reference point to dislocation with respect to the quantum dot base. In the calculation, the base diameter is set as constant which equals $20 \mathrm{~nm}$ and the height of the QD is $5 \mathrm{~nm}$. The substrate is large enough compared to the QD. In our simulation, the Descartes coordinate is adopted. The $x$ and $y$ axis correspond to the $[-1-10]$ and $[1-10]$ directions, respectively, while QD growth is along the [001] direction. The InAs quantum dot is assumed to be an ellipsoid shaped quantum dot which has been shown in Figure 1, respectively. Dislocation with line vector $[1-10]$ and the Burger's vector 


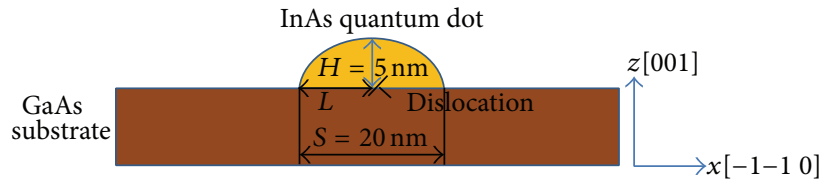

FIGURE 1: The cross section of ellipsoid shaped InAs quantum dots with GaAs substrate.

[01 - 1] for $60^{\circ}$ mixed dislocation is produced when the quantum dot was big enough $[15,16]$. The stiffness matrixes of InAs and GaAs materials in this case can be derived by tensor transformation [18] which has been shown in Tables 1 and 2 .

In order to confirm the accuracy of the Finite Element Method, Gatti et al. [19] have already compared the elastic field of a dislocated island as found by FEM methodology with the same result calculated via atomistic simulations. And the good qualitative and quantitative correspondence is evident. So we use the FEM in this work directly and have not compared our results with the ones calculated via atomistic simulations.

We first consider the strain sources in the InAs quantum dot. After the onset of misfit dislocation, the strain form lattice mismatch and dislocation segment are exit simultaneously in the quantum dot. So we set both of them to be the initial strains in InAs quantum dot. The strain obtained from lattice mismatch can be expressed as $\epsilon_{i j}^{0}=\left(\left(a_{\mathrm{QD}}-\right.\right.$ $\left.\left.a_{\mathrm{sub}}\right) / a_{\mathrm{QD}}\right) \delta_{i j}(i, j=x, y, z)$, where $a_{\mathrm{QD}}$ and $a_{\text {sub }}$ are the lattice constants of the QD and substrate, respectively. For the strain $\epsilon_{i j}^{\mathrm{dis}}$ from dislocation segment, nonsingular, selfconsistent formulas [20] are used to simulate the strain field of dislocation segment which makes the strain remain finite everywhere, including on the dislocation lines themselves.

We then move on to the strain sources in GaAs substrate. Because the substrate is big enough, the strain from lattice mismatch can be ignored totally. The initial strain in GaAs substrate only comes from the dislocation segment.

In the calculation, the positions of the dislocation are changed by adjust the strain analytical formulas of dislocation segment. By assigning the proper conditions and reaching convergence, the solver can find the solution for the stress and strain fields directly. The total elastic energy in a volume $V$ can be given by the well-known relation $[21,22]$ :

$$
\begin{gathered}
E=\frac{1}{2} \int\left(\sigma_{x x} \varepsilon_{x x}+\sigma_{y y} \varepsilon_{y y}+\sigma_{z z} \varepsilon_{z z}+2 \sigma_{x y} \varepsilon_{x y}\right. \\
\left.+2 \sigma_{x z} \varepsilon_{x z}+2 \sigma_{y z} \varepsilon_{y z}\right) d V .
\end{gathered}
$$

But because of the existence of the initial stresses in quantum dot and substrate, the total elastic energy in this model should be

$$
\begin{aligned}
E=\frac{1}{2} \int\left(\left(\varepsilon_{x x}-\epsilon_{x x}^{0}-\varepsilon_{x x}^{\mathrm{dis}}\right) \sigma_{x x}+\left(\epsilon_{y y}-\epsilon_{y y}^{0}-\epsilon_{y y}^{\mathrm{dis}}\right) \sigma_{y y}\right. \\
+\left(\epsilon_{z z}-\epsilon_{z z}^{0}-\epsilon_{z z}^{\mathrm{dis}}\right) \sigma_{z z}+2\left(\epsilon_{x y}-\epsilon_{x y}^{0}\right) \sigma_{x y} \\
\left.+2\left(\varepsilon_{x z}-\epsilon_{x z}^{0}\right) \sigma_{x z}+2\left(\varepsilon_{y z}-\epsilon_{y z}^{0}\right) \sigma_{y z}\right) d V
\end{aligned}
$$

TABLE 1: Stiffness matrix of InAs used in this work (unit Gpa).

\begin{tabular}{lccccc}
\hline 103.945 & 24.745 & 45.4 & 0 & 0 & 0 \\
24.745 & 103.945 & 45.4 & 0 & 0 & 0 \\
45.4 & 45.4 & 83.29 & 0 & 0 & 0 \\
0 & 0 & 0 & 39.6 & 0 & 0 \\
0 & 0 & 0 & 0 & 39.6 & 0 \\
0 & 0 & 0 & 0 & 0 & 18.945 \\
\hline
\end{tabular}

TABLE 2: Stiffness matrix of GaAs used in this work (unit Gpa).

\begin{tabular}{lccccc}
\hline 145.7 & 26.9 & 53.8 & 0 & 0 & 0 \\
26.9 & 145.7 & 53.8 & 0 & 0 & 0 \\
53.8 & 53.8 & 118.8 & 0 & 0 & 0 \\
0 & 0 & 0 & 59.4 & 0 & 0 \\
0 & 0 & 0 & 0 & 59.4 & 0 \\
0 & 0 & 0 & 0 & 0 & 32.5 \\
\hline
\end{tabular}

where the $\sigma_{i j}$ and $\varepsilon_{i j}(i, j=x, y, z)$ are the stress and strain calculated from the FEM solver; $\epsilon_{i j}^{0}$ and $\epsilon_{i j}^{\text {dis }}(i, j=x, y, z)$ are the initial strain from lattice mismatch and dislocation segment.

Compared with other ways, this method avoids discontinuity at the dislocation core and can be extended to any specific material in the conditions of any shaped quantum dots and any types of dislocations with some care.

\section{Results and Discussions}

In this section, we adopt the energy criterion to predict the preferential formation site of misfit dislocations for the InAs quantum dot with GaAs substrate. By using the model and method mentioned above, we calculate the stress and strain fields of the quantum dot system with interfacial and noninterfacial $60^{\circ}$ mixed dislocation segments located at different sites. The total energy of the quantum dot system can be obtained from (2).

Firstly, we calculated the strain energy of three different mixed dislocations which located at the island/QD interface, $0.5 \mathrm{~nm}$ and $1 \mathrm{~nm}$ above the interface. Variation of the energy, as a function of the "relative distance" in ellipsoid shaped InAs quantum dots, is shown in Figure 2(a). From the result, it is clear that the dislocations at interface are more energy favorable than the noninterfacial dislocations. It can explain the phenomenon that most of the misfit dislocations are located at the island/substrate interface. However, in some cases, the dislocations are observed located above the InAs/GaAs interface because of the surface oxidation of the material which resulted in the substrate surface moving down.

When the dislocation position along the QD growth direction is fixed, the most energy favourable position at the plane perpendicular to the QD growth direction is always at a "relative distance" 0.8 for the interfacial and noninterfacial mixed misfit dislocations. It means the positions near the edge of the quantum dot base are the energy favorable area for both interfacial and noninterfacial dislocations and 


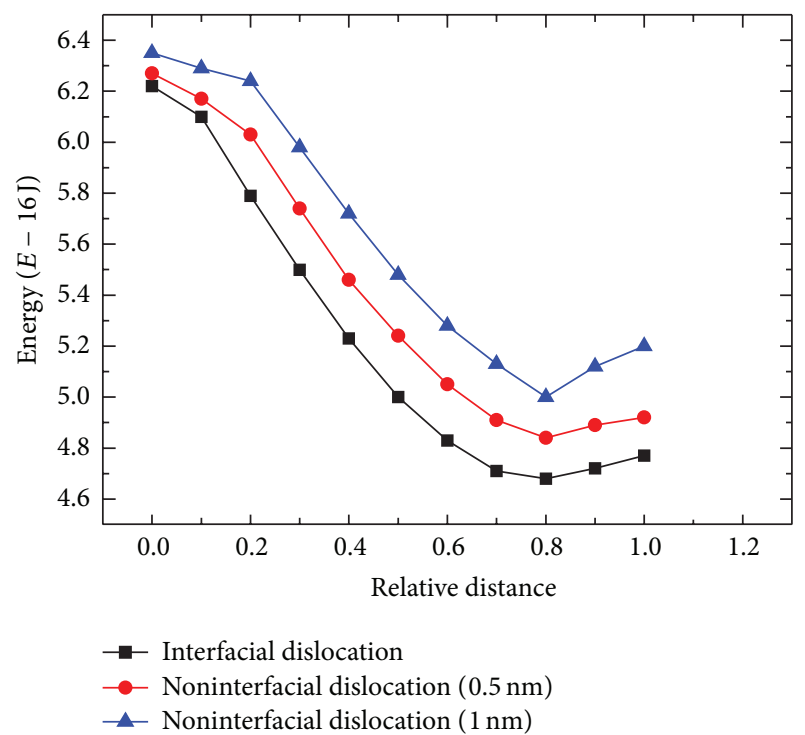

FIGURE 2: The relationship between energy and "relative distance" for $60^{\circ}$ mixed dislocations located at island/substrate interface (black line), $0.5 \mathrm{~nm}$ (red line), and $1 \mathrm{~nm}$ (blue line) above the island/substrate interface, respectively.

island/substrate interface has little impact on the preferential position of dislocations at the plane perpendicular to the QD growth direction. This phenomenon may be because of the relative position of the dislocation is only related with the type of dislocation and the shape of the QD. The experiment result of reference [12] can support our result partly. From the TEM picture in reference [12] (Figure 1), we can see that one dislocation is located at the position of relative distance $=0.8$.

In order to gain a clear idea of the most important factor which influences the dislocation preferential formation site of mixed dislocation at the plane perpendicular to the QD growth direction in InAs quantum dots, we decompose the $60^{\circ}$ mixed dislocation into two edge dislocation components with Burgers vector [110] and [00 - 1], respectively, and a screw dislocation component with Burgers vector [-110]. The relationship between energy and "relative distance" for the three dislocation components of interfacial and noninterfacial misfit dislocations are shown in Figures 3-5. From Figure 3, it is clear that the most energy favourable position for screw dislocation is located near the edges of the QD. So it is very easy to draw the conclusion that the screw dislocation component always tends to slip out of the QD and it has a positive influence on mixed dislocation nucleate at edge of the QD. But since the screw dislocation cannot relax the strain and stress in quantum dots, it has a small effect on the $60^{\circ}$ mixed dislocation preferential relative formation site. The main factors which influence the dislocation nucleation position are the two edge components. Owing to the fact that InAs quantum dot has a bigger lattice constant than GaAs substrate, compressive strain primarily exists in quantum dot; thus the two edge dislocation components must form at the positions which can relax the compressive strain in quantum dot most effectively. For the edge dislocation component with

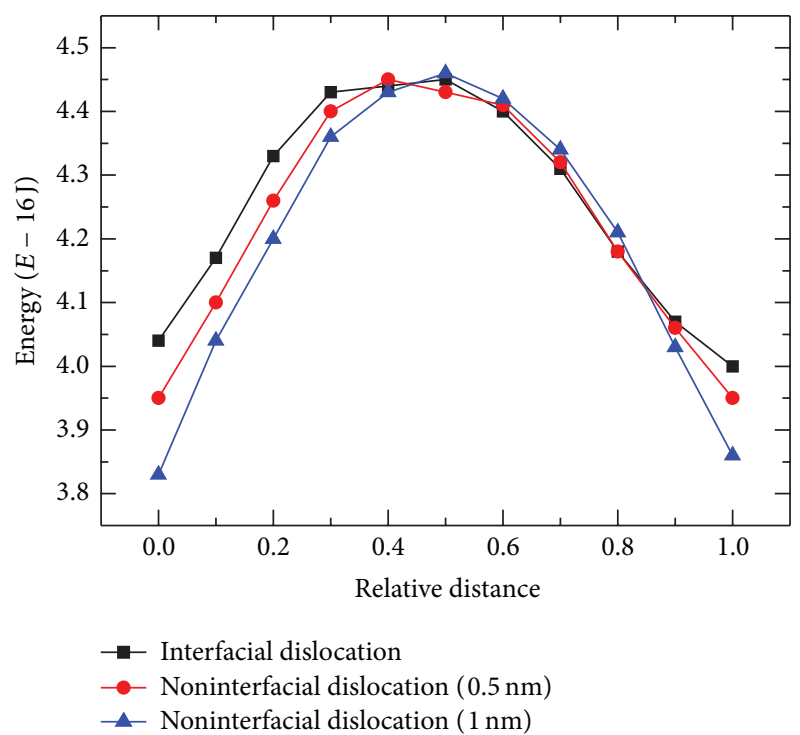

FIgURE 3: The relationship between energy and "relative distance" for screw dislocation component with Burgers vector [-110] located at island/substrate interface (black line), $0.5 \mathrm{~nm}$ (red line), and $1 \mathrm{~nm}$ (blue line) above the island/substrate interface, respectively.

Burgers vector [110] in Figure 4, it only exerts the tensile stress on quantum dot to balance out the compressive stress so that it firstly forms at the centre area of the island/quantum dot interface where the compressive stress reaches its maximum value. In the case of edge dislocation component with Burgers vector [00 - 1] in Figure 5, it preferentially nucleates at the right margin inside the quantum dot and it is because of that position the edge dislocation can relax the compressive strain inside the quantum dot most effectively and can exert no compressive strain on the quantum dot. We should also note that when the Burgers vector of this edge dislocation component becomes [001], the preferential nucleate site is at the left margin inside the quantum dot. So the $60^{\circ}$ mixed dislocation preferential nucleation site at the plane perpendicular to the QD growth direction depends on a compromise of the two edge dislocation component formation areas.

Based on the analysis above, the $60^{\circ}$ mixed dislocation will nucleate at the island/QD interface preferentially in common and positions near to the edge of the quantum dot base are the energy favorable areas for $60^{\circ}$ mixed dislocations. In some special cases, the misfit dislocations can locate above the island/substrate interface, but the preferential relative position at the plane perpendicular to the $\mathrm{QD}$ growth direction of the mixed dislocation position is still the same.

\section{Conclusion}

In this paper, using FEM, 3D models have been built to predict the preferential formation site of dislocations in InAs/GaAs quantum dot system in the framework of elastic anisotropy. Based on the dislocation position dependent model, we calculate the stress/strain field and total elastic 


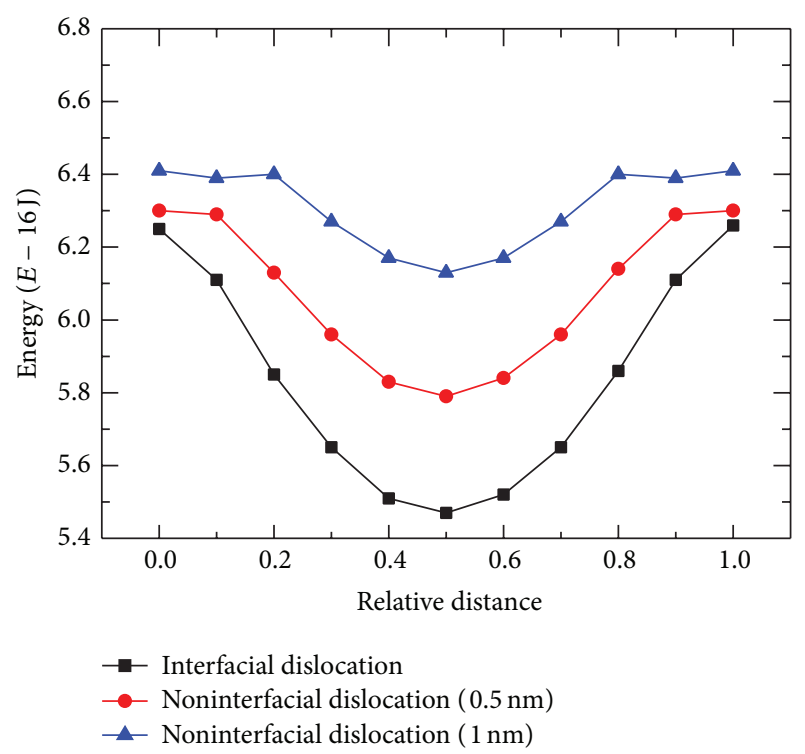

FIGURE 4: The relationship between energy and "relative distance" for edge dislocation component with Burgers vector [110] located at island/substrate interface (black line), $0.5 \mathrm{~nm}$ (red line), and $1 \mathrm{~nm}$ (blue line) above the island/substrate interface, respectively.

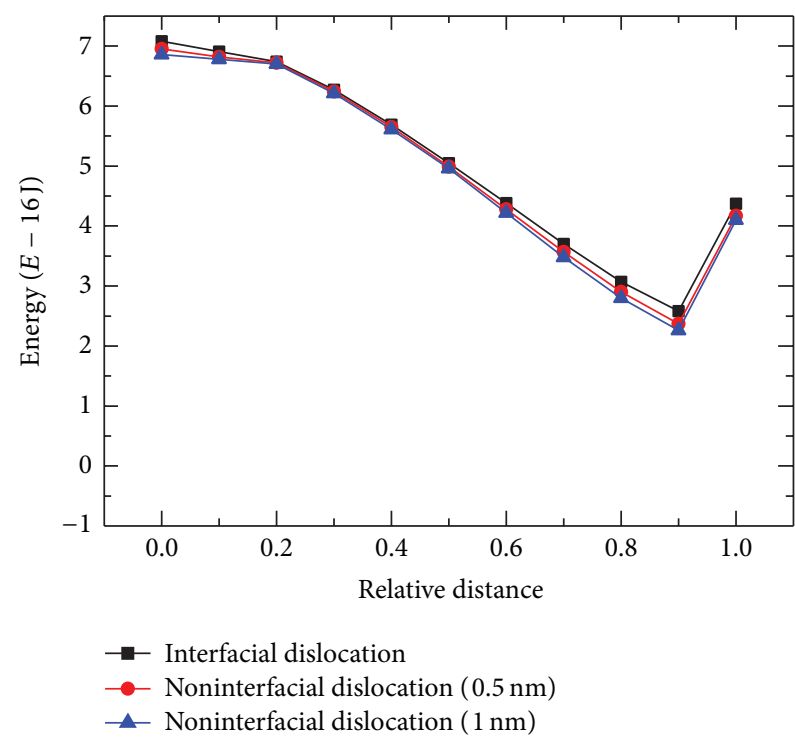

FIGURE 5: The relationship between energy and "relative distance" for edge dislocation component with Burgers vector [00 - 1] located at island/substrate interface (black line), $0.5 \mathrm{~nm}$ (red line), and $1 \mathrm{~nm}$ (blue line) above the island/substrate interface, respectively.

energy of the different types of dislocations in InAs/GaAs quantum dot system. From the calculation, we found that the $60^{\circ}$ mixed dislocation will nucleate at the island/QD interface preferentially in common. In some special cases, when the misfit dislocations located above the island/substrate interface, the preferential relative position of noninterfacial dislocation is the same as the interfacial one. Since once the dislocation position along the QD growth direction is fixed, the most important factors which influence the relative position of mixed misfit dislocation are the two edge dislocation components of the mixed dislocation rather than the island/substrate interface.

\section{Acknowledgments}

This Project was supported by the National High Technology Research and Development Program of China (Grant no. 2009AA03Z405), the National Natural Science Foundation of China (Grant nos. 60908028, 60971068, and 10979065), the Program for New Century Excellent Talents in University of China (NCET-10-0261), and the Chinese Universities Scientific Fund (no. 2011RC0402).

\section{Conflict of Interests}

The authors declare that there is no conflict of interests.

\section{References}

[1] V. M. Ustinov and A. E. Zhukov, "GaAs-based long-wavelength lasers," Semiconductor Science and Technology, vol. 15, p. R41, 2000.

[2] N. N. Ledentsov, A. R. Kovsh, A. E. Zhukov et al., "High performance quantum dot lasers on GaAs substrates operating in $1.5 \mu \mathrm{m}$ range," Electronics Letters, vol. 39, no. 15, pp. 1126-1128, 2003.

[3] L. Seravalli, P. Frigeri, G. Trevisi, and S. Franchi, "1.59 $\mu \mathrm{m}$ room temperature emission from metamorphic InAs/InGaAs quantum dots grown on GaAs substrates," Applied Physics Letters, vol. 92, no. 21, pp. 213104-213106, 2008.

[4] I. Tångringa, H. Q. Ni, B. P. Wu et al., " $1.58 \mu \mathrm{m}$ InGaAs quantum well laser on GaAs," Applied Physics Letters, vol. 91, no. 22, pp. 221101-221103, 2007.

[5] Y. M. Liu, Z. Y. Yu, and Y. Z. Huang, “The strain distributions and carrier's confining potentials of self-organized inas/gaas quantum dot," International Journal of Modern Physics B, vol. 20, no. 29, pp. 4899-4907, 2006.

[6] H. Ye, P. F. Lu, Z. Y. Yu, and L. H. Han, "Critical lateral dimension for a nanoscale-patterned heterostructure using the finite element method," Semiconductor Science and Technology, vol. 24, no. 2, Article ID 025029, 2009.

[7] Y. M. Liu, Z. Y. Yu, and X. M. Ren, "The strain relaxation of InAs/GaAs self-organized quantum dot," Chinese Physics B, vol. 18, no. 3, pp. 881-887, 2009.

[8] K. Yamaguchi, K. Yujobo, and T. Kaizu, "Stranski-Krastanov growth of InAs quantum dots with narrow size distribution," Japanese Journal of Applied Physics, vol. 39, no. 12, pp. L1245L1248, 2000.

[9] T. Manoa, K. Watanabea, S. Tsukamotoa, H. Fujiokab, M. Oshimab, and N. Koguchia, "Fabrication of InGaAs quantum dots on $\operatorname{GaAs}\left(\begin{array}{ll}0 & 0 \\ 1\end{array}\right)$ by droplet epitaxy," Journal of Crystal Growth, vol. 209, no. 2-3, pp. 504-508, 2000.

[10] T. Manoa, K. Watanabea, S. Tsukamotoa, H. Fujiokab, M. Oshimab, and N. Koguchia, "New self-organized growth method for InGaAs quantum dots on $\mathrm{GaAs}(001)$ using droplet epitaxy," Japanese Journal of Applied Physics, vol. 38, pp. 10091011, 1999.

[11] S. Zhou, Y. Liu, D. Wang et al., "The preferential formation site of dislocations in InAs/GaAs quantum dots," Superlattices and Microstructures, vol. 51, no. 1, pp. 53-61, 2012. 
[12] Z. B. Chen, W. Lei, B. Chen et al., "Can misfit dislocations be located above the interface of InAs/GaAs (001) epitaxial quantum dots?" Nanoscale Research Letters, vol. 7, article 486, 2012.

[13] Y. H. Kim, J. Y. Lee, Y. G. Noh, M. D. Kim, and J. E. Oh, "High-resolution transmission electron microscopy study on the growth modes of GaSb islands grown on a semi-insulating GaAs (001) substrate," Applied Physics Letters, vol. 90, p. 241915, 2007.

[14] Y. M. Liu, Z. Y. Yu, X. M. Ren, and Z. H. Xu, "Intensity modulation characters of orthogonally polarized HeNe lasers with different optical feedback level," Chinese Physics B, vol. 17, no. 2, p. 3471, 2008.

[15] Z. Mi, J. Yang, P. Bhattacharya, and D. L. Huffaker, "Selforganised quantum dots as dislocation filters: the case of GaAsbased lasers on silicon," Electronics Letters, vol. 42, no. 2, pp. 121123, 2006.

[16] J. Yang, P. Bhattacharya, and Z. Mi, "High-performance $\operatorname{In}_{0.5}$ $\mathrm{Ga}_{0.5}$ As/GaAs quantum-dot lasers on silicon with multiplelayer quantum-dot dislocation filters," IEEE Transactions on Electron Devices, vol. 54, no. 11, pp. 2849-2855, 2007.

[17] O. Brandt, K. Ploog, R. Bierwolf, and M. Hohenstein, "Breakdown of continuum elasticity theory in the limit of monatomic films," Physical Review Letters, vol. 68, no. 9, pp. 1339-1342, 1992.

[18] Y. M. Liu, Z. Y. Yu, X. M. Ren, and Z. H. Xu, "Intensity modulation characters of orthogonally polarized HeNe lasers with different optical feedback level," Chinese Physics B, vol. 17, no. 2, pp. 3471-3478, 2008.

[19] R. Gatti, A. Marzegalli, V. A. Zinovyev, F. Montalenti, and L. Miglio, "Modeling the plastic relaxation onset in realistic SiGe islands on Si(001)," Physical Review B, vol. 78, no. 18, Article ID 184104, 2008.

[20] W. Cai and A. Aresnlis, "A non-singular continuum theory of dislocations," Journal of the Mechanics and Physics of Solids, vol. 54, no. 3, pp. 561-587, 2006.

[21] H. Ye, P. Lu, Z. Yu, B. Jia, H. Feng, and Y. Liu, "Equilibrium critical size of coherent InSb/GaSb quantum dot," Physica E, vol. 42, no. 9, pp. 2402-2405, 2010.

[22] J. P. Hirth and J. Lothe, Theory of Dislocations, Wiley, New York, NY, USA, 1982. 

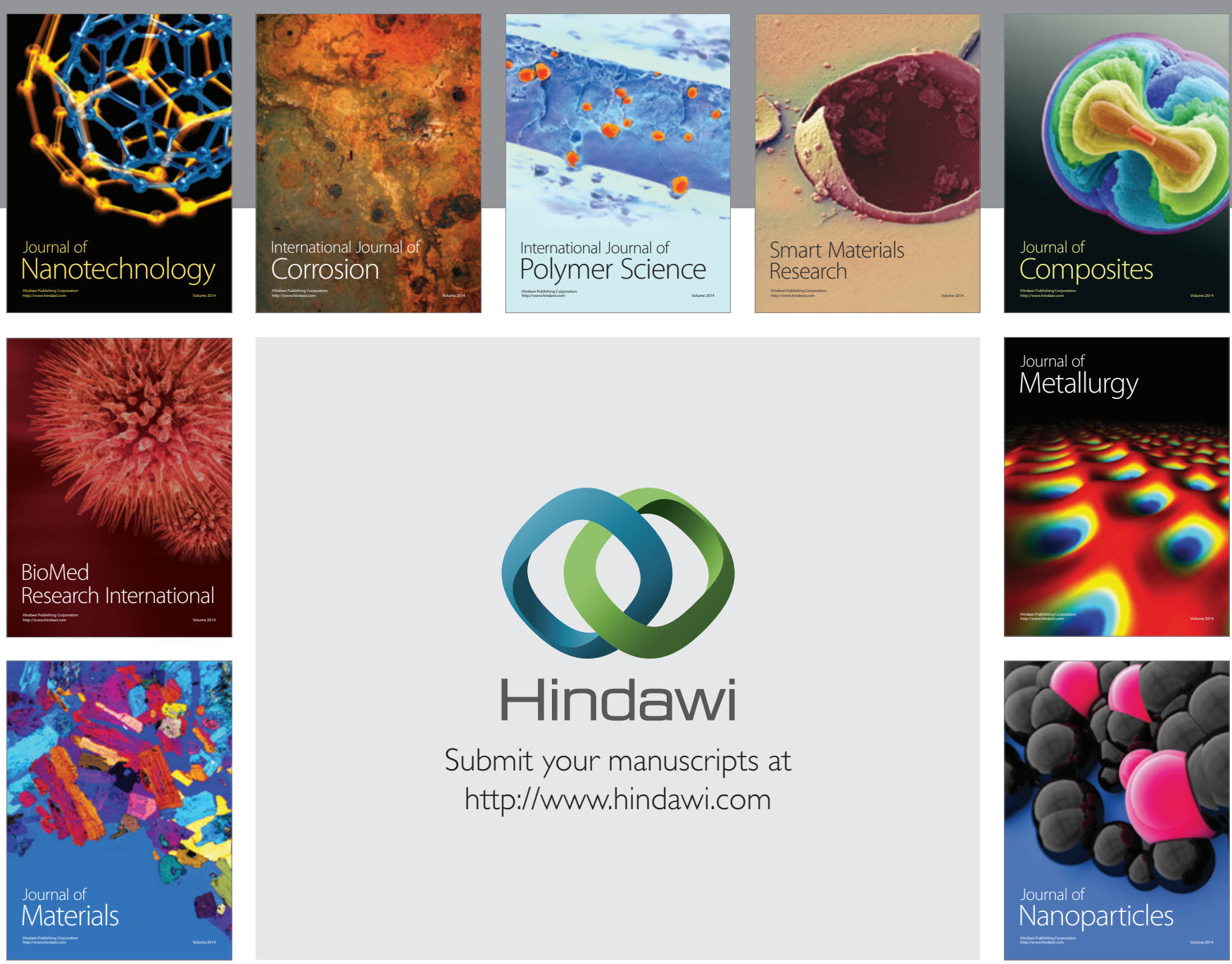

Submit your manuscripts at http://www.hindawi.com
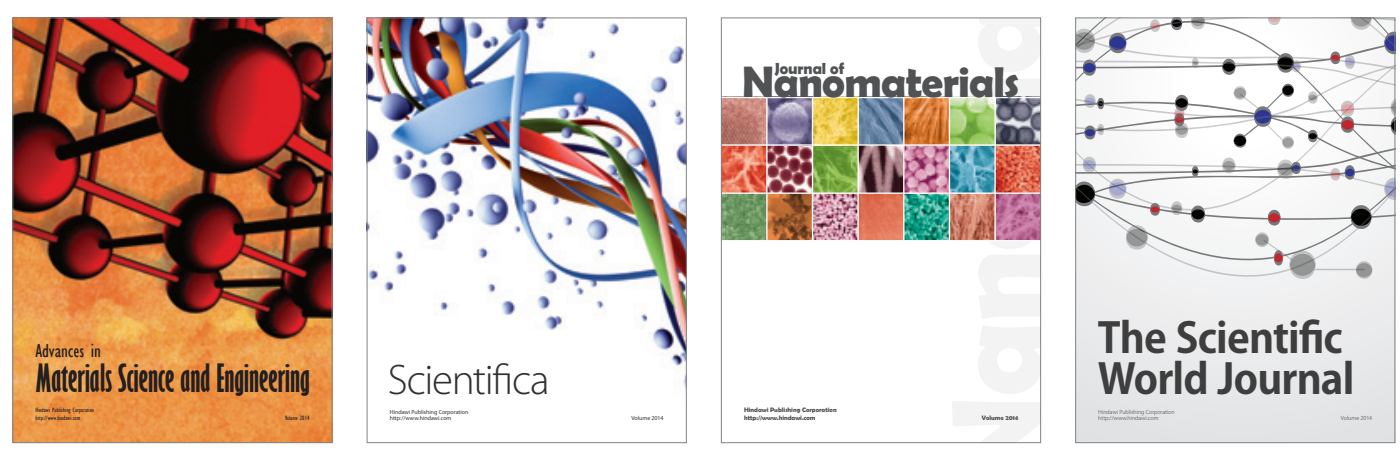

\section{The Scientific World Journal}
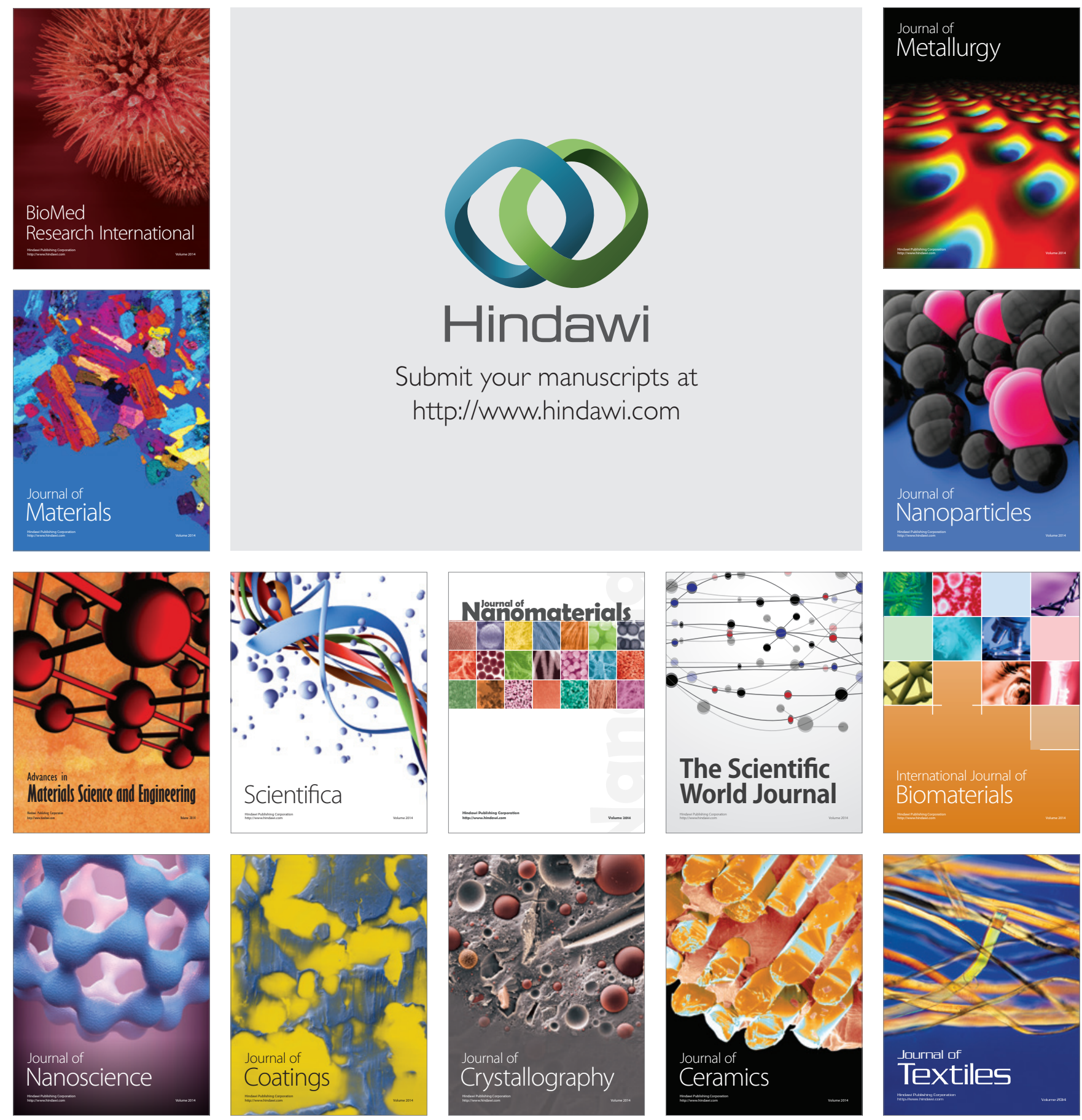